\title{
Ultra-thin gate oxides prepared by alternating current anodization of silicon followed by rapid thermal anneal
}

\author{
Yung-Chieh Chen, Chuang-Yuan Lee, Jenn-Gwo Hwu* \\ Department of Electrical Engineering, Room 446, National Taiwan University, Taipei, Taiwan 106, ROC
}

Received 28 December 2000; received in revised form 18 April 2001; accepted 20 April 2001

\begin{abstract}
An advanced method to prepare ultra-thin $(<30 \AA)$ anodic oxides is proposed. The voltage polarity applied to the silicon wafer is switched between a positive and a negative value periodically during anodic oxidation. It is belived that the more effective negative charges causing a barrier height for electron tunneling are generated in the oxide when a negative bias is applied to the wafer. In addition, the compensation effect of anion redistribution during voltage polarity switching contributes to eliminating the number of leakage paths in the oxide. Therefore, oxides prepared by this technique and followed by rapid thermal anneal exhibit lower leakage current and higher breakdown endurance than the conventional constant-voltage anodic oxides and the rapid thermal oxides do. In addition, various switching conditions are investigated to examine the effects of negative bias and the switching frequency upon the characteristics of oxides so that a better growth condition can be found to grow high-quality gate oxides. () 2001 Elsevier Science Ltd. All rights reserved.
\end{abstract}

Keywords: Gate oxide; Anodic oxidation; Alternating current

\section{Introduction}

As the metal-oxide-semiconductor field-effect transistor (MOSFET) dimension shrinks continuously, the gate oxide thickness is required to decrease to less than 3 $\mathrm{nm}$. In this regime, the oxide leakage current is dominant by a direct tunneling current that strongly depends on oxide uniformity and quality. It was found that better thickness uniformity leads to more optimistic projection of oxide reliability [1]. Therefore, many efforts have been paid to grow ultra-thin gate oxides with uniform thickness and improved reliability. Momose et al. have demonstrated the manufacturing feasibility of uniform $1.5 \mathrm{~nm}$ gate oxides with a standard deviation of around $0.13 \mathrm{~nm}$ [2]. In addition, the gate leakage current

\footnotetext{
* Corresponding author. Tel.: +886-2-2363-5251 ext.: 446; fax: +886-2-2367-1909.

E-mail address: hwu@cc.ee.ntu.edu.tw (J.-G. Hwu).
}

of MOSFETs was widely investigated to examine the reliability issues for sub-100 $\mathrm{nm}$ technology [3].

Among many approaches used to grow oxide films, anodic oxidation, or anodization, is featured as a room temperature process. Previous research focused on the electrochemical formation of the anodic oxide and its growth kinetics modeling [4-6]. However, since anodic oxide has the merit of less pinholes and good uniformity, anodic oxidation becomes a potential method to grow gate oxides in MOSFET devices [7]. Literature evidence shows that oxides prepared by anodic oxidation followed by rapid thermal nitridation exhibit higher quality than pure rapid thermal oxides due to the incorporation of nitrogen which relieves interfacial strain and suppresses interface trap generation [8]. With the advantages of anodic oxidation mentioned above, in this paper, a technique called "alternating current anodization of silicon" (ACAS) is proposed to meet the high-quality requirement of ultra-thin gate oxides. Conventionally, anodic oxidation has been carried out by applying constant voltage or constant current between the $\mathrm{Si}$ wafer 
and the Pt plate. Here, we switch the voltage polarity with reference to the $\mathrm{Pt}$ plate periodically instead of applying positive constant voltage to the wafer throughout the oxidation process. It is believed that ion redistribution and compensation effects occur during voltage polarity switching and make oxides more electrically uniform. Experimentally, it is proved that oxides prepared by this approach exhibit lower leakage current level and improved breakdown endurance the conventional oxides. Compared to previous research on anodic oxide around $50 \AA$ thick [8], we focus on oxide thickness less than $30 \AA$. This achievement is quite significant to the recent ultra-large scale integration technology in thin gate oxide preparation.

\section{Experimental}

This distinctive feature of the ACAS is that the voltage polarity applied to the wafer is switched positive and negative periodically, instead of only applying constant positive voltage in the conventional anodic oxidation. Therefore, experiments are designed to discuss the effects of the voltage polarity switching upon the electrical performance of the ACAS oxides. These effects are examined in two respects. One is the negative field duration and the other is the switching frequency. The detailed voltage profiles applied to the silicon wafer designed for these two experiments will be described later. Here we make an introduction to the fabrication procedures for testing devices used in the whole work.

Fig. 1(a) shows the experimental setup system for ACAS. To switch the voltage polarity, a dual-tracking DC power supply and a relay are used. The dualtracking DC power supply has two outputs of which channel 1 is set to a positive value and channel 2 to a negative one. The switch in the relay is controlled by a voltage, $V_{\text {ctrl }}$, which is provided by a programmable (a)

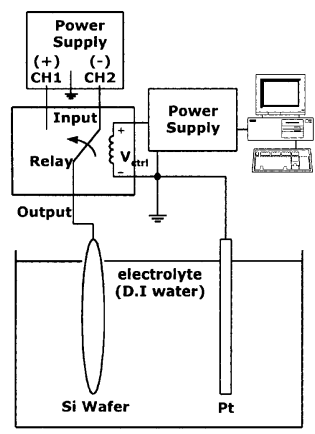

(b)
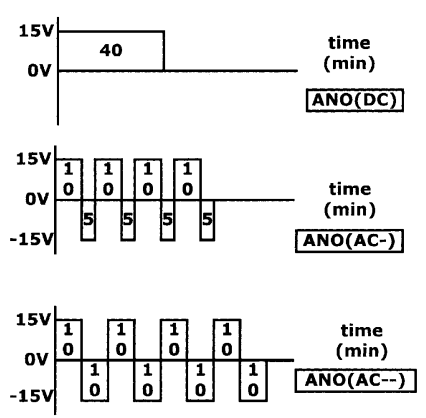

Fig. 1. (a) Schematic diagram of experimental setup for ACAS. (b) Designed voltage profiles applied to the silicon to examine the effects of negative field duration. power supply. For the relay used in this system, if $V_{\text {ctrl }}=0 \mathrm{~V}$, the relay is normally OFF and the output voltage equals $V_{\mathrm{CH} 2}$; if $V_{\text {ctrl }}=10 \mathrm{~V}$, the relay is enabled so that its output voltage switches to $V_{\mathrm{CH} 1}$. With this design, the switching condition is absolutely controlled by the power supply whose output voltage value can be determined by a computer program.

Testing devices used in this study are metal-oxidesemiconductor (MOS) capacitors fabricated on 3-in., P-type $(100)$ silicon wafers. After the standard RCA cleaning, the native oxide on the wafer is removed using diluted HF solution before the oxidation. The oxidation is carried out by both anodic oxidation (ANO) in deionized water and rapid thermal oxidation (RTO) in $\mathrm{O}_{2}$ ambient (400 Torr) at $850^{\circ} \mathrm{C}$ for $15 \mathrm{~s}$. The RTO samples are used for comparison. It is noted that the distance between the Pt plate (cathode) and the wafer (anode) is kept at $5 \mathrm{~cm}$ during anodization. Next, rapid thermal post-oxidation anneal is treated to all samples in $\mathrm{N}_{2}$ ambient (400 Torr) at $850^{\circ} \mathrm{C}$ for $15 \mathrm{~s}$ to densify the oxides. The oxide thickness is then measured by ellipsometry with refractive index $n=1.46$. Following this, the aluminum film with a thickness around $3000 \AA$ is evaporated on the oxides as the metal gate and the photolithography is performed to make $150 \times 150 \mu \mathrm{m}^{2}$ square patterns. Aluminum films are evaporated again to the backside of the wafers after removing back oxides to form back contacts. Time-zero dielectric breakdown (TZDB) measurement is first done to observe the leakage current level and oxide breakdown field. In a TZDB test, a negative gate voltage with $0.1 \mathrm{~V} / \mathrm{s}$ staircase ramp is applied to a MOS capacitor. The oxide breakdown occurs when the measured current exceeds $50 \mu \mathrm{A}$. The breakdown voltage $V_{\mathrm{BD}}$ is recorded and the breakdown field $E_{\mathrm{BD}}$ is obtained from $V_{\mathrm{BD}}$ divided by oxide thickness. Time-dependent dielectric breakdown (TDDB) measurement also performed to investigate the oxide endurance to the hot carrier injection. In this test, a constant field is applied to a MOS capacitor till the current exceeds $50 \mu \mathrm{A}$. Time to breakdown $t_{\mathrm{BD}}$ is recorded. Gate injection is adopted throughout this work.

\section{Results and discussion}

\subsection{Effects of negative field duration}

The voltage profiles designed to analyze the effects of the negative bias hold for different time duration during anodization are shown in Fig. 1(b). Oxides grown with constant positive voltage only is denoted by AN$\mathrm{O}(\mathrm{DC})$, and the oxides grown by ACAS are denoted by ANO(AC-) and ANO(AC--). The number of "-" means the length of time for which the negative voltage is applied. The final oxide thicknesses of samples ANO(DC), ANO(AC-), ANO(AC--) and RTO are 27, 26, 27 and 


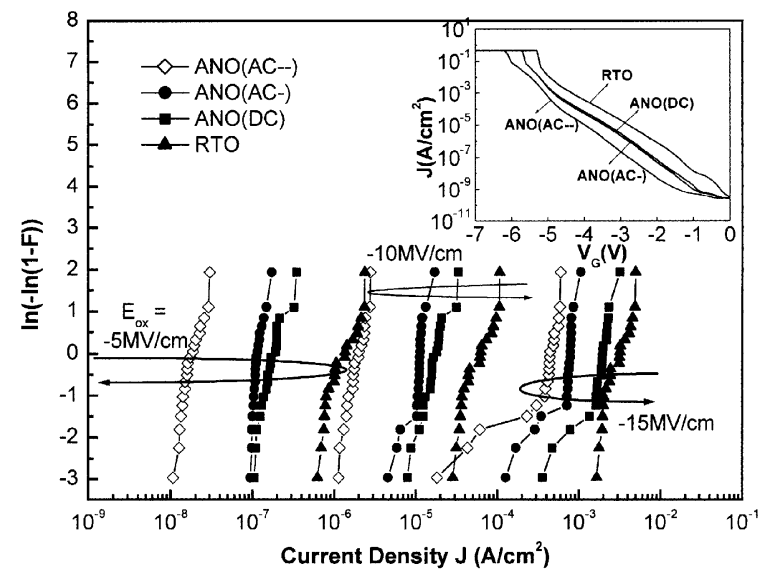

Fig. 2. Typical current density vs. gate voltage $(J-V)$ characteristics and Weibull plots of the gate leakage current densities under oxide fields of $-5,-10$ and $-15 \mathrm{MV} / \mathrm{cm}$ for MOS capacitors with $\mathrm{ANO}(\mathrm{AC}-\mathrm{-}), \mathrm{ANO}(\mathrm{AC}-), \mathrm{ANO}(\mathrm{DC})$ and RTO oxides.

$25 \AA$, respectively. One can see that even the process time of $\mathrm{ANO}(\mathrm{AC}-\mathrm{-}), 80 \mathrm{~min}$, is twice as much as that of $\mathrm{ANO}(\mathrm{DC}), 40 \mathrm{~min}$, the oxide thickness of $\mathrm{ANO}(\mathrm{AC}--)$ is almost the same with that of $\mathrm{ANO}(\mathrm{DC})$. This may be given as evidence that oxidation does not occur while a negative voltage is applied to the wafer.

Fig. 2 shows the gate current density vs. gate voltage $(J-V)$ characteristics of MOS capacitors fabricated by $\mathrm{ANO}(\mathrm{DC})$, ANO(AC-), ANO(AC--) and RTO. Measurements were performed on 20 capacitors for each condition. The Weibull plots of the gate leakage current densities under three oxide field stresses, $-5,-10$ and $-15 \mathrm{MV} / \mathrm{cm}$, are also shown in Fig. 2. The flat-band voltage of each sample is calculated by $C V$ method according to the $C V$ characteristics as demonstrated in Fig. 3. The flat-band voltage has been taken into account in calculating the oxide field as mentioned above. In addition, the Weibull distribution of TZDB and TDDB under $-16 \mathrm{MV} / \mathrm{cm}$ for these four samples is shown in Fig. 4. It is noted that 20 capacitors are measured for each sample in these breakdown endurance tests. Obviously, for ANO(DC) oxides, the leakage currents are smaller and the breakdown endurance is higher than RTO oxides. ANO(AC-) oxides have slightly bit better characteristics than $\mathrm{ANO}(\mathrm{DC})$ oxides do. Specifically, ANO(AC--) oxides show even lower leakage current level and higher breakdown endurance than that of the other three oxides. It is believed that three are some pretrapped negative oxide charges in the anodic oxides. These negative charges are hydrogenrelated defects and increase the barrier height between metal and oxide so that the gate leakage current is retarded for anodic oxides. Besides, the anodic oxide has fewer pinholes and good uniformity so that the break-

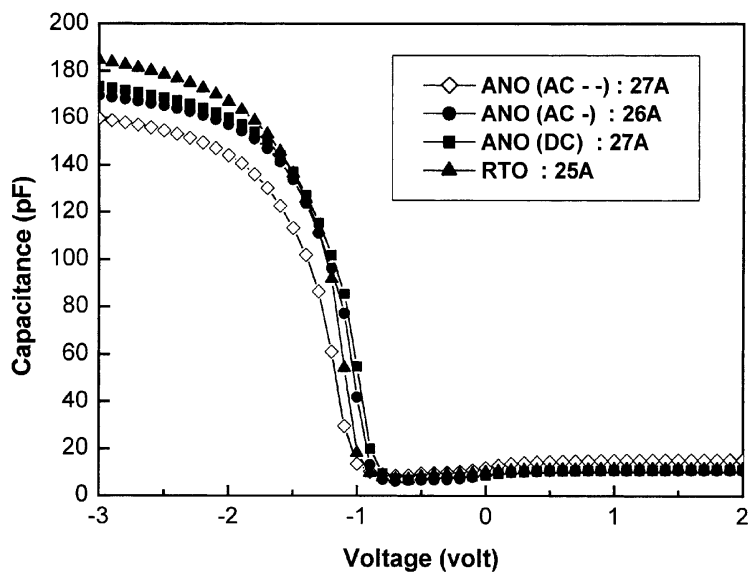

Fig. 3. High-frequency capacitance-voltage $(C-V)$ characteristics of MOS capacitors with $\mathrm{ANO}(\mathrm{AC}--)$, $\mathrm{ANO}(\mathrm{AC}-)$, AN$\mathrm{O}(\mathrm{DC})$ and RTO oxides.

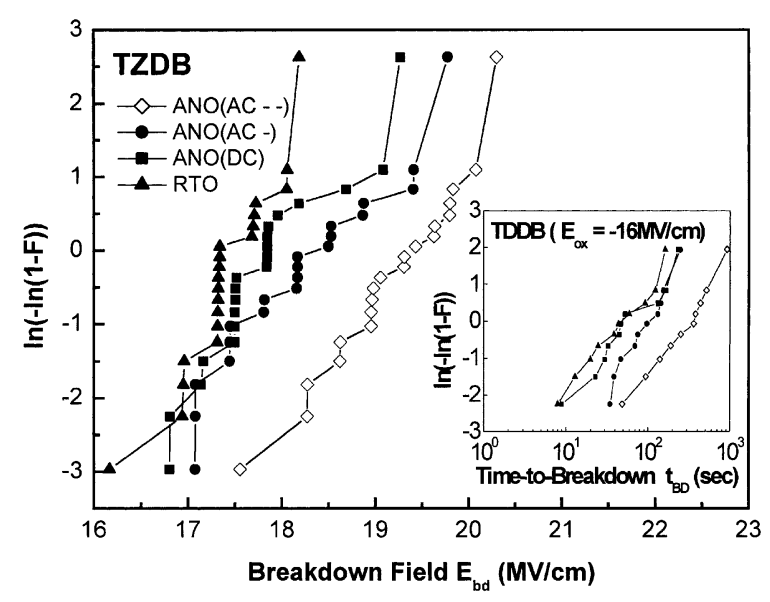

Fig. 4. Weibull plots of the TZDB and TDDB tests under -16 $\mathrm{MV} / \mathrm{cm}$ of MOS capacitors with $\mathrm{ANO}(\mathrm{AC}--)$, ANO(AC-), $\mathrm{ANO}(\mathrm{DC})$ and RTO oxides.

down endurance is improved. For ever better characteristics of $\mathrm{ANO}(\mathrm{AC})$ oxides, it is supposed that some oxide leakage paths are being repaired during voltage polarity switching. Moreover, more effective negative oxide charges are supposed to appear if the duration of negative bias applied is longer. These negative charges raise up the oxide energy barrier in the metal/oxide interface and thus suppress the electron current flow.

To further prove the above assumption, the barrier height at the metal- $\mathrm{SiO}_{2}$ interface for each sample is calculated using the classical Fowler-Nordheim (FN) tunneling current formula $[9,10]$ as follows.

$\frac{J}{F^{2}}=C \mathrm{e}^{-\beta / F}$ 
where $J$ is the current density, $F$ is the field across the oxide, and the pre-exponent $C$ and slope $\beta$ are given by

$C=\frac{q^{3} m}{16 \pi^{2} \hbar m_{\mathrm{ox}} \phi_{\mathrm{B}}}=1.54 \times 10^{-6} \frac{m}{m_{\mathrm{ox}}} \frac{1}{\phi_{\mathrm{B}}} \quad\left(\mathrm{A} / \mathrm{V}^{2}\right)$,

and

$$
\begin{aligned}
\beta & =\frac{4}{3} \frac{\left(2 m_{\mathrm{ox}}\right)^{1 / 2}}{q \hbar} \phi_{\mathrm{B}}^{3 / 2} \\
& =6.83 \times 10^{7}\left(\frac{m_{\mathrm{ox}}}{m}\right)^{1 / 2} \phi_{\mathrm{B}}^{3 / 2} \quad(\mathrm{~V} / \mathrm{cm}),
\end{aligned}
$$

where $q$ is the electron charge, $m$ and $m_{\mathrm{ox}}$ the electron mass in free space and in the oxide respectively, $h$ is Planck's constant, and $\phi_{\mathrm{B}}$ is the barrier height expressed in electron volts, between the gate electrode and the oxide.

Using a value of $\left(m_{\mathrm{ox}} / m\right)=0.39$, barrier height $\phi_{\mathrm{B}}$ can be calculated from the slope of $\ln \left(J F^{-2}\right)$ vs. $F^{-1}$ plot. Fig. 5 shows this plot for the four kinds of oxides. As the value written in the figure, $\phi_{\mathrm{B}}$ is 4.19, 4.1, 3.94 and 2.95 $\mathrm{eV}$ for the ANO(AC--), ANO(AC-), ANO(DC) and RTO oxide, respectively. That is, anodic oxides prepared by ACAS methods have the highest barrier height, followed by the constant-voltage anodic oxide, and the barrier height for the RTO oxide is the lowest. This result provides good explanation for the lowest leakage current level of the ANO(AC--) oxide. In other words, the highest leakage current level of RTO oxide is due to the smaller value of its barrier height.

\subsection{Effects of switching frequency}

The effects of the frequency of the voltage polarity switching during anodic oxidation will be studied to get better oxide growth condition for ACAS. The profiles of the voltage applied to the silicon wafer designed for this purpose are shown in Fig. 6. The constant voltage an-

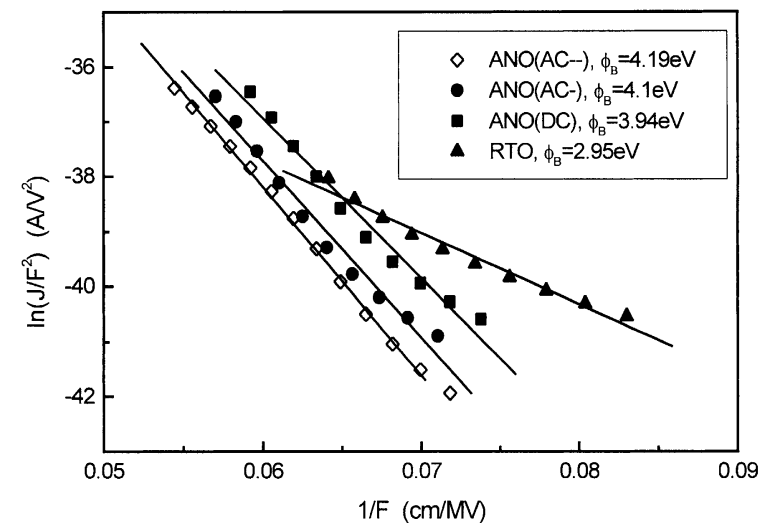

Fig. 5. The FN plots for $\mathrm{ANO}(\mathrm{AC}-\mathrm{-}), \mathrm{ANO}(\mathrm{AC}-), \mathrm{ANO}(\mathrm{DC})$ and RTO oxides.
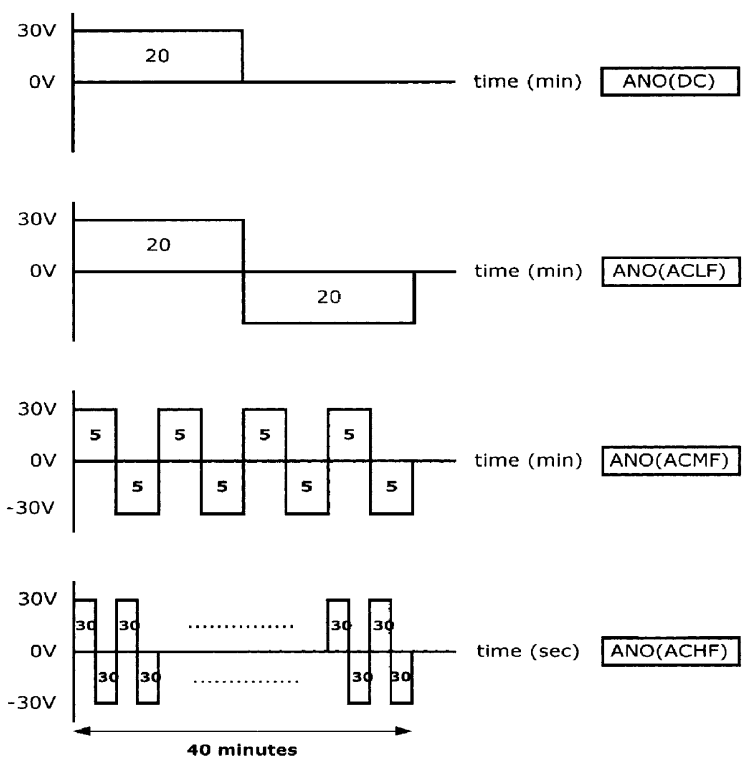

Fig. 6. Designed voltage profiles applied to the silicon to examine the effects of switching frequency.

odic oxide is denoted by $\mathrm{ANO}(\mathrm{DC})$, and the other oxides prepared by ACAS with lower to higher frequencies described in the figure are denoted ANO(ACLF), AN$\mathrm{O}(\mathrm{ACMF}), \mathrm{ANO}(\mathrm{ACHF})$, respectively. Note that the voltage sweep is raised from 15 to $30 \mathrm{~V}$ to save the oxidation time. The oxide thicknesses are 21, 21, 24 and 22 A, respectively.

Evaluation of the electrical properties of these four oxides is accomplished by the same testing procedures used in Section 3.1. The insert of Fig. 7. shows the gate

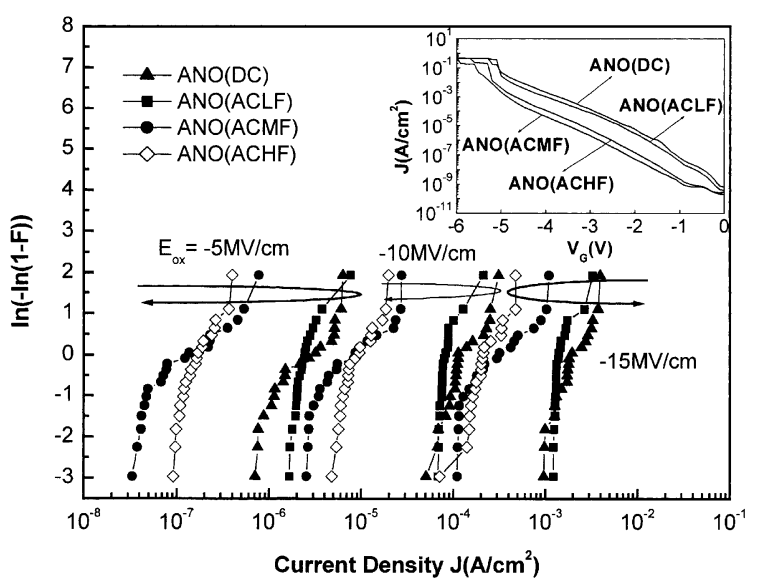

Fig. 7. Typical current density vs. gate voltage $(J-V)$ characteristics and Weibull plots of the gate leakage current densities under oxide fields of $-5,-10$ and $-15 \mathrm{MV} / \mathrm{cm}$ for MOS capacitors with $\mathrm{ANO}(\mathrm{ACHF}), \mathrm{ANO}(\mathrm{ACMF}), \mathrm{ANO}(\mathrm{ACLF})$ and ANO(DC) oxides. 
current density vs. gate voltage $(J-V)$ characteristics of MOS capacitors with the gate oxides grown by four different conditions mentioned above. The Weibull plots of the gate leakage current density under three oxide fields, $-5,-10$, and $-15 \mathrm{MV} / \mathrm{cm}$, are also shown in Fig. 7. From this figure, one can conclude that the leakage current of the ANO(DC) oxide is still the largest among the four. Actually the voltage polarity is switched once from positive to negative for the ANO(ACLF) oxide, but its current is a little smaller than $\mathrm{ANO}(\mathrm{DC})$, which may be caused by the effect of negative bias applied discussed before. A distinct decrease of the leakage current occurs in both ANO(ACMF) and ANO(ACHF) oxides. Again, it is observed that oxides prepared by ACAS methods have smaller gate current than conventional anodic oxides. This result can also be proven by calculating the barrier height at the metal/oxide interface using Eq. (3). The plots of $\left(J F^{-2}\right)$ vs. $F^{-1}$ are shown in Fig. 8. The calculated barrier heights are 4.1, 4.15, 3.27 and $2.99 \mathrm{eV}$ for $\mathrm{ANO}(\mathrm{ACHF}), \mathrm{ANO}(\mathrm{ACMF}), \mathrm{AN}-$ $\mathrm{O}(\mathrm{ACLF})$ and $\mathrm{ANO}(\mathrm{DC})$, respectively. These values demonstrated that equivalent negative charges, which increase the barrier height for electron tunneling, exist more in $\mathrm{ANO}(\mathrm{AC})$ oxides than in $\mathrm{ANO}(\mathrm{DC})$ oxides. The difference between the calculated barrier height of $2.99 \mathrm{eV}$ for ANO(DC) here and that of $3.94 \mathrm{eV}$ in Section 3.1, may result from distinct field distribution causing varied negative charge location. Furthermore, compared to the ANO(ACHF) oxide, the lower current level of the ANO(ACMF) oxide is supposed to be due to its thicker oxide thickness. Taking the oxide breakdown field (Fig. 9) into consideration, it is still believed that ANO(ACHF) oxides exhibit smaller leakage current than ANO(ACMF) oxides do, especially under highoxide field stress.

The Weibull plots of TZDB and TDDB tests under $-17 \mathrm{MV} / \mathrm{cm}$ are also shown in Fig. 9. The ANO(ACMF)

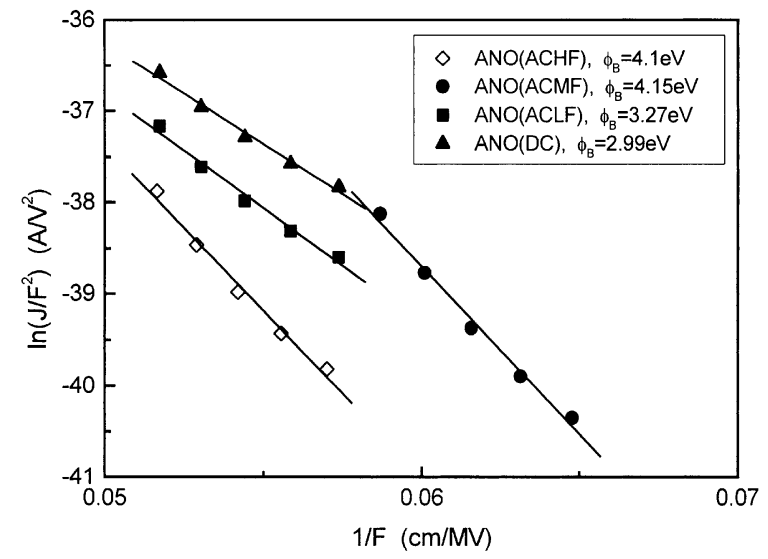

Fig. 8. The FN plots for ANO(ACHF), ANO(ACMF), AN$\mathrm{O}(\mathrm{ACLF})$ and $\mathrm{ANO}(\mathrm{DC})$ oxides.

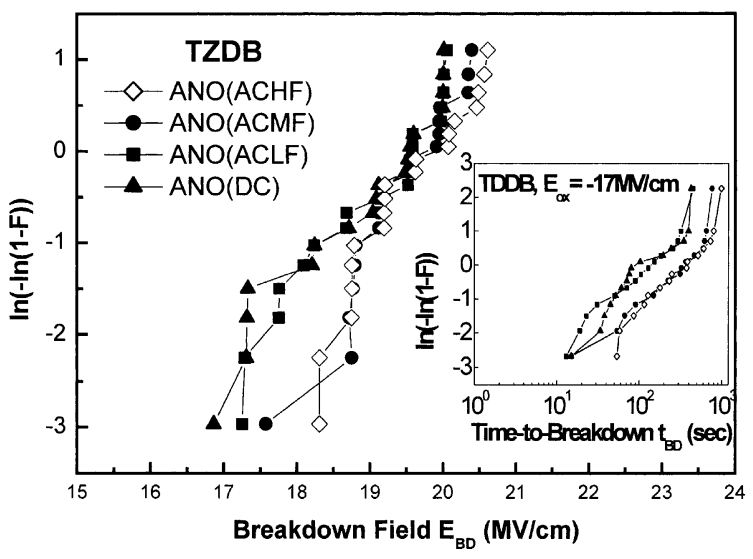

Fig. 9. Weibull plots of the TZDB and TDDB tests under -17 $\mathrm{MV} / \mathrm{cm}$ of MOS capacitors with ANO(ACHF), ANO(ACMF), $\mathrm{ANO}(\mathrm{ACLF})$ and $\mathrm{ANO}(\mathrm{DC})$ oxides.

and $\mathrm{ANO}(\mathrm{ACHF})$ oxides have longer time-to-breakdown under electrical stress than the ANO(ACLF) and ANO(DC) oxides. The results of this test tells that oxide interfacial properties are improved so that oxide reliability is enhanced for both ANO(ACMF) and AN$\mathrm{O}(\mathrm{ACHF})$.

As a preliminary conclusion, under a fixed growth time, if voltage polarity switches with a frequency higher to some extent, the ACAS oxides show lower leakage current level and greater breakdown endurance. This is because that if the switching frequency is increased, the oxide local leakage paths can be selected and repaired by the anions with greater probability during the reoxidation process while the voltage polarity is switched from negative to positive. That is, the oxide structure is more uniform with this treatment.

\subsection{Growth model for alternating current anodization of silicon}

In the previous parts, it is observed that the oxides grown by ACAS have better properties such as lower leakage current level and improved breakdown endurance. It is believed that the compensation effect and the anion redistribution contribute to fixing the leakage paths and defects in the oxide during ACAS. From this point of view, a simple growth model describing the mechanism of ACAS is presented as follows.

During anodic oxidation, water dissociates into $\mathrm{H}^{+}$ and $(\mathrm{OH})^{-}$when a voltage drop is generated between the $\mathrm{Pt}$ plate and the $\mathrm{Si}$ wafer. The $\mathrm{H}^{+}$ions will move to the cathode and the $(\mathrm{OH})^{-}$ions to the anode. If the Pt plate is grounded (set as $0 \mathrm{~V}$ ) throughout the processes and a positive voltage is applied to the wafer, the $(\mathrm{OH})^{-}$ will dissolve into the wafer and react with the silicon to form silicon dioxide. However, due to the inherent 
non-uniform electrical field between the $\mathrm{Pt}$ and $\mathrm{Si}$, the growth rate of each local oxidation path is not uniformly distributed, and the interfacial $\mathrm{Si}-\mathrm{O}$ bonding structure is not so perfect over the entire oxide film. Therefore, some defects or leakage paths occur in some local area of the oxide. When the anodization process is terminated, these leakage paths left in the oxide provide possible tunneling paths for the carriers injected to the oxide as the oxide becomes an MOS structure. Larger leakage currents will accelerate the oxide breakdown so that the oxide reliability is degraded.

Now if the voltage of the wafer is switched to a negative polarity, the $(\mathrm{OH})^{-}$ions will retreat to the $\mathrm{Pt}$ plate, and $\mathrm{H}^{+}$ions will move to the wafer. During this period of time, forming of the anodic oxide is suspended. When the voltage of the wafer is switched again to a positive value, the $\mathrm{H}^{+}$ions will move back to the $\mathrm{Pt}$ plate, and the $(\mathrm{OH})^{-}$ions will transport toward the silicon and the oxidation process restarts. At the moment of the polarity switching, all the $(\mathrm{OH})^{-}$ions reselect their forward directions. It is thus more probable for the anions to move directly or migrate over the oxide film to reach these leakier paths. With larger ionic current flow, oxide growth rate is increased or defects could be repaired locally through the leaky paths. This phenomenon is regarded as the compensation to the nonuniform distribution of oxide thickness and quality. Repeating this procedures to switch the voltage polarity, it provides enough time and greater probability for anions to reduce the number of much leakier paths in the oxide. This argument could be used to explain the effect of switching frequency in Section 3.2. That is, oxides prepared by ACAS with higher switching frequency exhibit better characteristics than that of oxides prepared by ACAS with lower switching frequency or constant-voltage anodic oxidation.

\section{Conclusion}

In this paper, the anodic oxidation is studied for growing ultra-thin gate oxides. In addition to the con- ventional constant-voltage anodic oxidation, a brand new technique named ACAS is also developed. The experimental results show that the ACAS oxides exhibit lower leakage current level and improved breakdown endurance than the constant-voltage anodic oxides and rapid thermal oxides do. Besides, the switching conditions during ACAS, including effects of the negative field duration and the switching frequency, are also discussed by performing several experiments. It is preliminary found that the oxide quality is improved with longer negative field duration and higher switching frequency. In conclusion, ACAS has been demonstrated to provide a feasible and reproducible approach to grow highquality oxide films with improved performance and reliability.

\section{Acknowledgements}

The authors would like to thank the National Science Council of the Republic of China for supporting this work under contract no. NSC89-2215-E-002-042.

\section{References}

[1] Weir BE, Silverman PJ, Alam MA, Baumann F, Monroe D, Ghetti A, Bude JD, Timp GL, Hamad A, Oberdick TM, Zhao NX, Ma Y, Brown MM, Hwang D, Sorsch TW, Madic J. IEDM Tech Dig 1999:437.

[2] Momose HS, Nakamura S, Ohguro T, Yoshitomi T, Morifuji E, Morimoto T, Katsumata Y, Iwai H. IEEE Trans Electron Dev 1998;45:691.

[3] Iwai H, Momose HS. IEDM Tech Dig 1998;163.

[4] Jain GC, Prasad A, Chakravarty BC. J Electrochem Soc 1979;126:89.

[5] Ghowsi K, Gale RJ. J Electrochem Soc 1989;136:867.

[6] Sharma SK, Chakravarty BC, Singh SN, Das BK, Parashar DC, Rai J, Gupta PK. J Phys Chem Solids 1989;50: 679.

[7] Revesz AG. J Electrochem Soc 1967;114:629.

[8] Jeng MJ, Hwu JG. J Appl Phys Lett 1996;69:3875.

[9] Lenzlinger M, Snow EH. J Appl Phys 1969;40:278.

[10] Weinberg ZA. J Appl Phys 1982;53:5052. 